\title{
Frontières
}

\section{LEONETTI, Jean, Vivre ou laisser mourir. Respecter la vie, accepter la mort, Paris, Éditions Michalon, 2005, 139 p.}

\section{Pierre-Olivier Hudon}

Volume 18, numéro 1, automne 2005

URI : https://id.erudit.org/iderudit/1074323ar

DOI : https://doi.org/10.7202/1074323ar

Aller au sommaire du numéro

\section{Éditeur(s)}

Université du Québec à Montréal

\section{ISSN}

1180-3479 (imprimé)

1916-0976 (numérique)

Découvrir la revue

Citer ce compte rendu

Hudon, P.-O. (2005). Compte rendu de [LEONETTI, Jean, Vivre ou laisser mourir. Respecter la vie, accepter la mort, Paris, Éditions Michalon, 2005, 139 p.]

Frontières, 18(1), 73-73. https://doi.org/10.7202/1074323ar d'utilisation que vous pouvez consulter en ligne.

https://apropos.erudit.org/fr/usagers/politique-dutilisation/ 
LEONETTI, Jean

\section{Vivre ou laisser mourir \\ Respecter la vie, accepter la mort}

Paris, Éditions Michalon, 2005, 139 p.

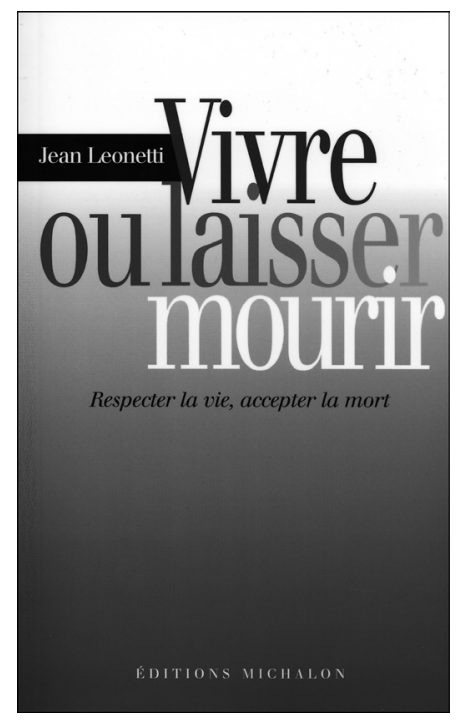

"Je vous demande le droit de mourir." Cette demande formulée par Vincent Imbert, un jeune homme de 21 ans tétraplégique, muet et presque aveugle, résume à elle seule la problématique abordée par Jean Leonetti dans Vivre ou laisser mourir. Respecter la vie, accepter la mort. Médecin, maire d'Antibes et député des Alpes-Maritimes, Leonetti a présidé la mission d'information sur l'accompagnement de la fin de vie créée en octobre 2003. Dans son essai, il expose les différents enjeux relevés lors de cette mission, qui a mené à une loi votée à l'unanimité en novembre 2004.

Dans le premier chapitre, "Les sociétés ont la mort qu'elles méritent» (p. 17 à 36), Leonetti expose le contexte dans lequel s'inscrit la démarche de législation sur l'accompagnement de fin de vie. Au cours de la seconde moitié du $X X^{e}$ siècle, l'événement du mourir est passé d'une expérience signifiante, religieuse et familiale à l'insignifiance qui afflige la vie elle-même quand elle est prise en charge par une escouade de spécialistes en robe blanche. Ce faisant, la mort n'est plus le point culminant de la vie d'un individu mais la conséquence pratique de l'impuissance de la médecine devant la maladie. Devant ce que Leonetti appelle la peur de mal mourir, la peur de mourir seul, à l'hôpital, dans la souffrance, et devant les témoignages de ceux qui veulent reprendre leur mort en main, le débat est lancé: peut-on choisir sa mort, ou plutôt peut-on choisir le moment de sa mort?

Les enjeux soulevés par cette question sont résumés dans le titre du deuxième chapitre, "La liberté ou la vie» (p. 37 à 51). Leonetti interroge d'abord la liberté à l'œuvre dans le choix de la mort. Dans quelle mesure un individu choisit-il librement de mourir? Il est vrai que l'acte de se tuer soi-même n'est pas un acte criminel. Même si le suicide est fortement condamné socialement, un individu demeure libre de se tuer si tel est son souhait. Dans un contexte où l'intégrité physique est malmenée par la maladie et la souffrance, le choix de s'enlever la vie ne peut s'effectuer que dans une liberté partielle. À la question de la liberté succède celle de la valeur sacrée de la vie humaine. Dans quelles conditions la vie cesse-t-elle d'avoir son caractère inestimable? Ici intervient la notion de dignité humaine, concept dont la définition est difficile à poser. Leonetti fait ressortir le fait que la dignité humaine s'établit toujours par rapport au regard d'autrui, que ce soit celui du médecin en devoir ou celui de la famille affligée par la souffrance d'un proche. Car c'est dans le regard d'autrui que la personne malade se saisit de la gravité de son état et qu'elle établit pour elle-même l'état de sa dignité, lequel orientera son choix entre la lutte contre la maladie ou l'abandon dans la mort. Cependant, entre ces deux réponses se trouvent différentes avenues à considérer dans le choix de la mort sans douleur.

Ces avenues sont présentées dans le troisième chapitre, "La mort douce» (p. 53 à 71). Leonetti y discute tour à tour des gestes de l'euthanasie, du suicide assisté, du refus de l'acharnement thérapeutique et du traitement par soins palliatifs. L'euthanasie est définie comme étant l'exécution anticipée d'une mort pratiquée dans l'intérêt du patient. Dans la pratique de l'euthanasie, Leonetti souligne le danger que l'intérêt du tiers exécutant la sentence en vienne à supplanter l'intérêt du patient. Le suicide assisté se définit comme la décision de se donner la mort par l'intermédiaire d'un tiers. Le contexte dans lequel la décision de la mort se prend est discuté dans le second chapitre. Par la suite, il est question de I'acharnement thérapeutique, une notion que Leonetti définit comme «une perte de lucidité de l'équipe médicale qui refuse de considérer que le traitement appliqué est inutile» (p. 65) L'ambiguïté ici réside dans la justification a posteriori du traitement. Ainsi, I'acharnement thérapeutique apparaît au moment où le traitement échoue. Dans le cas d'une guérison, l'acharnement thérapeutique devient un miracle de la médecine. Les soins palliatifs, quant à eux, sont envisagés lorsqu'un patient ne répond plus aux soins curatifs. Il s'agit d'une médecine centrée sur le confort du patient pour que son passage vers la mort se fasse de la meilleure façon possible. Pour Leonetti, ces quatre gestes doivent être compris dans la loi sur l'accompagnement de fin de vie.

Dans l'avant-dernier chapitre de Vivre ou laisser mourir, "Le malade, le médecin et le juge» ( $p .73$ à 109), Leonetti explore le rôle des différents intervenants qui seront à I'œuvre dans l'application d'une loi sur l'accomplissement de fin de vie. D'abord, même s'il doit s'en remettre au médecin dans sa maladie, le patient a des droits: celui de savoir et celui de choisir, peu importe que ce choix se fasse entendre par la voix d'un acte légal préalablement rédigé ou par la voix d'une personne mandatée. Le médecin, quant à lui, doit se concentrer sur l'intérêt du patient tout en exerçant son devoir médical. II doit traiter tantôt pour guérir, tantôt pour atténuer les souffrances. Entre les deux types de traitement, il y a le danger de l'erreur médicale et des conséquences légales qui s'en suivent. Ainsi, la proposition de loi sur l'accompagnement de fin de vie se doit de contenir toutes les nuances possibles afin de protéger l'intérêt du patient et l'action du médecin et pour éviter les excès des différents intervenants. Leonetti situe les excès possibles comme les deux pôles d'un même cercle. D'un côté, il y a le danger d'une utilisation abusive de doses de morphine mortelles, dispensées volontairement pour donner la mort. De l'autre, il y a la crainte que les doses de morphines nécessaires à la cessation de la douleur ne soient pas données par crainte de I'homicide involontaire. Comme le souligne le titre du dernier chapitre, il s'agit de rédiger "une loi pour faire vivre et laisser mourir» ( p. 111 à 121). Faire vivre, c'est ne pas administrer la mort à l'insu du patient, ni à l'insu de la famille. Laisser mourir, c'est condamner l'obstination déraisonnable et laisser au patient le droit de refuser un traitement jugé nécessaire à sa survie. Au centre de ce choix entre la vie et la mort, il y a le patient, accablé par la maladie, à qui l'on accorde tout de même la responsabilité de faire un choix éclairé à travers le voile de la souffrance.

L'essai de Leonetti fait très bien ressortir les enjeux de la démarche de législation sur l'accompagnement de fin de vie. Le texte est très bien rédigé, ponctué de statistiques et d'exemples qui explicitent les ambiguïtés au cœur de la question de la mort sans douleur. Le livre de Leonetti est un excellent outil pour qui cherche à y voir plus clair sur le sujet.

\section{Pierre-Olivier Hudon}

